\title{
Evaluación de políticas y dación de cuentas, requisitos para una adecuada gobernabilidad territorial a partir de una nueva planificación; combinando política y geografía ${ }^{1}$
}

Evaluation of Policies and Accountability, Requirements for an Appropriate Territorial Governability from a New Planning by Combining Politics and Geography

Joaquín Farinós Dasín

Para citar este artículo, utilice el nombre completo así:

Farinós Dasí, J. (2014). Evaluación de políticas y dación de cuentas, requisitos para una adecuada gobernabilidad territorial a partir de una nueva planificación; combinando política y geografía. Perspectiva Geográfica, 19 (2), 331-356

\section{Resumen}

Una adecuada gobernabilidad del territorio es un factor clave para lograr un desarrollo justo, democrático y en dignidad. Para ello se consideran elementos decisivos la reinterpretación de la labor de gobierno, con renovadas prácticas de gobernanza más democrática y participada, la incorporación del territorio en la agenda política y la recuperación del espacio de vida en aplicación y

\footnotetext{
1 Este artículo corresponde al proyecto de investigación "Del gobierno a la gobernanza y gobernabilidad efectiva del territorio: guías para un nuevo desarrollo territorial"; Ministerio de Economía y Competitividad. Plan Nacional de I+D+i 2008-2011. Subprograma de Proyectos de Investigación Fundamental. Gobierno de España.

2 Geógrafo. Doctor en Geografía por la Universitat de València. Catedrático de Análisis Geográfico Regional en la citada Universidad. Miembro fundador del IIDL de la UVEG. Autor de diversos libros y artículos que reflejan el interés por la planificación del desarrollo territorial, la geografía política regional y el diseño y evaluación de políticas públicas desde las escalas de proximidad. Ponente y profesor invitado en diversos congresos, seminarios y programas de varias universidades españolas, europeas y latinoamericanas. Investigador responsable y coordinador en diversos proyectos nacionales e internacionales.
} 
desarrollo del principio de subsidiariedad. Muchas de estas cuestiones forman parte del acervo común y representan un potencial y atractivo campo de desarrollo profesional de la geografía. El texto analiza las relaciones entre gobierno, gobernanza y gobernabilidad, tendiendo puentes entre geografía y política, lo que facilita una nueva aproximación a las funciones de planificación y gestión del potencial territorial existente. La ordenación territorial representa uno de los campos predilectos de buena gobernanza. Se argumenta que la dimensión simbólica de la gobernanza (como proceso) resulta más decisiva que la material (estructura o precondición) para una adecuada gobernabilidad de los territorios que conduzca a la cohesión y el bienestar (buen vivir).

Palabras clave: cohesión territorial, evaluación de impacto territorial, gobernabilidad, gobernanza democrática, planificación territorial estratégica integral.

\section{Abstract}

Right governability constitutes itself a key factor for a just, more democratic and dignified development. Key issues to achieve it are re-interpretation of government actions by including new more democratic and collaborative governance practices, the incorporation of territory in the political agenda, the recovery of living space and development of the subsidiarity principle. Many of these issues are part of a geographical 'acquis' or background, and they represent a potential as well as an attractive professional field of application for geographers. This paper analyzes government, governance and governability relationships, and builds bridges between geography and politics. That facilitates a new and more useful approach to planning and management of available territorial potential. Spatial planning is one of the signs of good governance practices. It is argued that the symbolic dimension of governance (understood as a process) is more decisive than material (as a structure or pre-condition) in order to achieve right governability leading to cohesion and welfare ('buen vivir').

Keywords: territorial cohesion, territorial impact assessment, governability, democratic governance, comprehensive strategic spatial planning. 


\section{Introducción}

La tesis que se defiende en este artículo es que la gobernabilidad del territorio, entendida como la aceptación y el cumplimiento de las reglas del Estado de derecho por parte de todos los actores presentes en un espacio y en igualdad de condiciones, es la meta final y la garantía de un pretendido desarrollo territorial sostenible. Un desarrollo que se entiende justo, democrático, en dignidad y derechos, más allá del simple crecimiento económico y de una interpretación simplista y unívoca del mismo como modernización.

Para ello se analizan las relaciones entre gobierno, gobernanza y gobernabilidad. Desde una perspectiva no solo politológica sino, de forma complementaria con esta, territorial y geográfica. Esta combinación permite un acercamiento renovado, entendemos que más potencial y útil, a la tarea de la planificación territorial. Una función o política pública preocupada por el uso racional (ya no solo bajo un modelo de racionalidad 'perfecta' -tecnocrática y solo al alcance de los expertos- sino también discursiva/deliberativa entre la multiplicidad de actores) de los recursos y la atractividad territoriales (el denominado 'capital territorial'), al objeto de procurar una mejor calidad de vida de los ciudadanos y lograr un mayor equilibrio entre territorios y regiones (principio y objetivo de la cohesión territorial).

Planificación u ordenación del territorio representa uno de los campos, si no el campo predilecto, en donde se pueden aplicar los principios de buena gobernanza. Este se interpreta en este caso más como conjunto de rutinas o procesos mediante los que se adoptan, implementan y evalúan políticas, planes, programas y proyectos (lo que podemos denominar su componente 'simbólico'), que como estructura o precondición (modelo de Estado o de administración) que las sustentan (su componente 'material'). Nuestra hipótesis, a tenor de una amplia explotación de bibliografía y documentación gris de análisis de experiencias, así como de los resultados obtenidos en trabajos e investigaciones previas, que aquí se sintetizan, es que las primeras resultan más cruciales que las segundas. Sin embargo son las que han recibido mayor atención, con las sucesivas reformas del aparato del Estado y las administraciones, sin acabar de lograr los resultados esperados en materia de desarrollo territorial sostenible ni en los impactos territoriales, ambientales y sociales que se generan. Ello sugiere la necesidad de reorientar el foco hacia la dimensión simbólica de la gobernanza a la hora de poder garantizar mejor la pretendida gobernabilidad de los territorios. 
De este modo, y partiendo de la gobernabilidad territorial como meta, se analizarán algunos determinantes de la misma, tales como la cultura política, la participación pública y el sistema de toma de decisiones y de seguimiento y evaluación (acciones típicas de gobernanza democrática). En este punto el texto centra su atención en algunas cuestiones clave, tales como el necesario ejercicio de transparencia y apertura en materia de política territorial o políticas con impacto territorial (lo que nos devuelve al tema de la información, la observación y la inteligencia territoriales, todas elementos indispensables para una verdadera y útil planificación deliberativa); las posibilidades reales y ventajas comparativas de una planificación estratégica de tipo colaborativo; cómo poder incorporar el territorio y las políticas territoriales en la agenda política, promoviendo una nueva relación entre la academia y los expertos con los tomadores de decisiones; y el papel que las evidencias, una adecuada información, diagnóstico y evaluación territoriales, juegan para ello.

La evaluación territorial o de los impactos territoriales de las políticas, resulta una cuestión de gran trascendencia para hacer útiles no solo los instrumentos de planificación del desarrollo territorial sostenible, sino también su legitimidad; aspecto que mucho tiene que ver con las posibili- dades de una gobernabilidad territorial real (legitimidad por resultados democráticamente decididos) $\mathrm{y}$, finalmente, las posibilidades de modelos de desarrollo alternativos, más eficientes, más justos, más sustentables y en dignidad.

La actual situación de crisis financiera y social en el mundo más desarrollado clama por una revisión de los modelos y prácticas de desarrollo imperantes hasta la fecha y de la forma en que se han producido, y sirve de ejemplo para aquellos nuevos países que tratan de experimentar y desarrollar nuevas sendas, como la del llamado 'buen vivir'. La reinterpretación de la labor de gobierno, con renovadas prácticas de gobernanza más democrática y participada, la incorporación del territorio (con su carácter transdisciplinar, más que interdisciplinar, y de sistema complejo) en la agenda política, y la recuperación del espacio de vida (el nivel local) en aplicación y desarrollo del principio de subsidiariedad (empleando las relaciones y síntesis multiescalares como método), son todos elementos considerados como factores coadyuvantes para una adecuada gobernabilidad de los territorios. Muchas de estas cuestiones forman parte del acervo común de la geografía, que en estas líneas se defiende, tiene en este asunto, su entendimiento, aproximación y formulación de alternativas (desde la conceptualiza- 
ción y diagnóstico, hasta la formulación de sistemas e indicadores de seguimiento y evaluación, el soporte a la selección de alternativas, la evaluación y seguimiento), un potencial y atractivo campo de desarrollo profesional al que mirar con especial atención e interés.

\section{Buen gobierno y gobernanza del territorio ¿de qué estamos hablando?}

En algún texto precedente (Farinós, 2008) ya referíamos que la gobernanza era un concepto de dudosa procedencia y sospechosa intención, especialmente por el posible riesgo de desregulación y subordinación del poder público frente a los actores privados, el mercado y la sociedad civil. Se trata de un riesgo real al que se vienen enfrentando no pocos Estados nacionales que no cuentan con una sociedad civil suficientemente potente y organizada ni con unos mecanismos de control y dación de cuentas bien desarrollados. En estas circunstancias la sociedad civil se muestra incapaz de hacer frente tanto a algunos efectos del mercado como, también a veces, de los actores institucionales (la gobernación); actúen ambos por separado o en connivencia. Esta es la razón fundamental, más allá de consideraciones y motivaciones ideológicas, de la desconfianza e incluso desdén sobre la idea y las prácticas de lo que se ha dado en llamar nueva gobernanza.

A pesar de ello, o precisamente por ello, no podemos negar la mayor; esto es, que viejos modelos y fórmulas reglamentistas, burocráticas y dirigistas no han dado los resultados esperados. Pero tampoco la desregulación y la confianza ciega en el mercado en pos del crecimiento; más aún en tiempos de profunda crisis financiera, económica y, lo que es peor, ética, aquello a lo que paradójicamente nos hemos visto conducidos por aquel. La labor necesaria para poder afrontar esta situación apunta en una única dirección de tres vías: facilitar los mecanismos de control y dación de cuentas; procurar nuevos enfoques, métodos y rutinas para producir los instrumentos de planificación y su posterior implementación con el afán de producir los efectos deseados, dado que hasta la fecha, leyes, políticas y planes (resultando imprescindibles) en demasiadas ocasiones se demuestran insuficientes; y desarrollar una nueva cultura política y territorial, a la que justamente pretenden contribuir las dos anteriores.

No debe olvidarse que la utilidad de las nuevas formas de gobernanza es que estas representan una innovación en la forma de hacer gobierno, tratando de superar las limitaciones a las que aquel se enfrenta y que le im- 
piden lograr una gestión efectiva de la política pública. Por lo tanto, gobierno y gobernanza, más que antagónicos, resultan complementarios, si no consecutivos -en forma de espiral ascendente- (Farinós, 2008). Tampoco cabe olvidar que las nuevas formas de gobernanza no garantizan que exista gobernabilidad; se trata de una condición necesaria, pero no suficiente ${ }^{3}$. Esto nos devuelve a la necesidad del Estado de derecho, pero también de la conciencia ciudadana y del desarrollo de una adecuada cultura política y territorial que sostenga la actividad y el control de la sociedad civil sobre cualquier proceso que afecte al bien o interés general (más allá de una participación banal) como medio para ofrecer propuestas de solución a pro-

\footnotetext{
3 Por gobernanza se entiende, de acuerdo con la primera acepción del diccionario de la Real Academia de la Lengua Española "Arte o manera de gobernar que se propone como objetivo el logro de un desarrollo económico, social e institucional duradero, promoviendo un sano equilibrio entre el Estado, la sociedad civil y el mercado de la economía". Implica por tanto una ampliación del número de actores presentes en la toma de decisiones políticas, más allá de los actores institucionales de la gobernación. En cuanto a los principios que caracterizarían la buena gobernanza, por su simplicidad y carácter básico se destacan los cinco que refiere el Libro blanco de la gobernanza de la UE (2001): (hacer transparentes y comunicar adecuadamente las decisiones), (implicando a los ciudadanos en la elaboración y aplicación de las políticas), (clarificando el papel de cada actor en el proceso de toma de decisiones para que asuma su responsabilidad, de acuerdo con el principio de proporcionalidad), (para poder mejorarla, las decisiones se tomarán en el nivel y momento más pertinentes, en aplicación del principio de subsidiaridad y proporcionalidad), (entre las distintas políticas, actuaciones y administraciones responsables; curiosamente uno de los principios a los que se presta menor atención y al que se entrega con mayor énfasis la planificación territorial integral).

Sobre las diferencias entre gobierno, gobernanza y gobernabilidad vid. Prats (2005a, p. 133 y ss.).
}

blemas desestructurados (no a los que ya la tienen -estructurados-). De esta nueva cultura política y territorial también forma parte tender puentes entre academia/expertos y tomadores de decisiones, y entre estos y la sociedad. Ello supone transitar, de la y la a la, para llegar finalmente a la (Buroway, 2005, citado por Ferrao 2010). ${ }^{4}$

La sociedad, la política y las élites no han logrado desarrollar de forma efectiva proyectos de carácter colectivo, estructurados relacionalmente y dirigidos estratégicamente en interés (general/común) del territorio. En gran parte debido a la falta de unas instituciones de gobierno permeables, demasiado preocupadas las más de las ocasiones en no perder el control tanto de su proceso de elaboración como sobre todo de los efectos de los mismos. Ello ha acabado por conducir a dos extremos: a los conflictos territoriales o a la simple indiferencia social y política (Davoudi, Farinós, Paül \& De Vries, 2009; Queirós, 2009; Seixas, 2008; Wassenhoven, 2008). Las instituciones públicas deben entender que los grupos de actores involucrados en la planificación estratégica de su espacio de vida son capaces de generar un nuevo orden que va regenerándose a sí mismo (Queirós, 2009, p. 17).

\footnotetext{
4 Sobre esta cuestión pueden verse, entre otros Chilvers \& Evans (2009). Para un mayor detalle sobre el papel y las relaciones de la academia en la puesta en práctica de planes y estrategias territoriales vid. Healey (2008).
} 
Ambos extremos acabados de referir se encuentran alejados precisamente de lo que se entiende por buen gobierno del territorio: la forma de organizar un consenso entre los distintos actores para promover soluciones satisfactorias para todos ellos. Con este nuevo modelo de gestión y de decisión sobre los asuntos públicos se trata de acordar una estrategia compartida para el futuro del territorio entre todos los niveles de poder y actores implicados/concernidos, que deciden sobre su futuro deseado pero también, y esto es lo más importante, sobre la forma en que cada uno de ellos se compromete a contribuir en su consecución.

Así pues, la gobernanza territorial hace referencia a un nuevo entendimiento de la acción pública y de sus estructuras organizativas, de la forma en que los territorios de un Estado son administrados y las políticas aplicadas. Supone la emergencia y puesta en práctica de innovadoras formas (compartidas) de planificación y de gestión de las dinámicas territoriales, respaldadas por múltiples actores que comparten unos objetivos y que conocen y asumen cuál es el papel que deben desempeñar para poder lograrlos.

Es aquí donde tiene perfecto acomodo un nuevo estilo de planificación territorial estratégica (Albrechts, 2004, 2006; Albrechts, Healy
\& Kunzmann, 2003; Healy, 2004, 2006; Salet \& Faludi, 2000); más concretamente la planificación estratégica de segunda generación, también llamada por algunos autores 'nueva planificación territorial estratégica' (Albrechts, 2009; Pascual, 2007). Se diferencia fundamentalmente de la de primera generación en que los nuevos planes se realizan incorporando la evaluación mediante la definición, en el mismo momento de elaboración del plan, de indicadores de seguimiento que permiten su evaluación de forma continuada. También se caracteriza por tratar de fortalecer los elementos de gobernanza democrática tales como el tejido de redes sociales, una verdadera implicación ciudadana y la asociación público-privada. Una planificación de nuevo cuño, con un nuevo estilo y sobre todo unas nuevas prácticas y rutinas.

\section{De la gobernanza a la goberna- bilidad... el largo camino}

Junto a la primera definición de gobernanza que se refería en la primera nota a pie de página, el diccionario de la Real Academia de la Lengua Española en una segunda acepción la define como "Acción y efecto de gobernar o gobernarse". En ella parece prevalecer, atendiendo al orden y primera parte del enunciado, el papel principal que se otorga al Estado (en 
cualquiera de sus niveles de decisión: administración general -central o federal-, regional y local; todo es Estado) sobre los otros dos componentes (la sociedad civil y el mercado). Se correspondería esta interpretación con la confusión como sinónimos de "gobernanza" y "buen gobierno", un argumento que hasta la fecha ha sido defendido por muchos autores que en sus trabajos abogaban por un marcado enfoque neoinstitucionalista (que comparte quien suscribe). Este posicionamiento se debía a los recelos que la gobernanza, término de sospechosa procedencia y dudosa reputación, despertaba, como se ha comentado.

La opción de una gobernanza reforzada, otorgando al Estado el papel motor y preeminente en la relación a tres con el mercado y la sociedad civil, no es la que se defendía en otros contextos (vid. por ejemplo el informe de 2003 del canadiense 'Institute on Governance'). La gobernanza, que explícitamente se interpreta como cosa distinta del (buen) gobierno, incluye las tradiciones, instituciones y procesos que determinan cómo es ejercido el poder, cómo los ciudadanos intervienen o pueden intervenir y cómo se toman las decisiones en asuntos de interés general, y también la forma en que actúan los mercados. En este último caso, desde el punto de vista de la gobernanza, por el comportamiento de los ciudadanos como consumidores exigentes, recla- mando a las empresas responsabilidades de orden social, medioambiental y territorial (algo que podríamos bautizar como Responsabilidad Territorial Colaborativa ), y que estas aprovechan para la fidelización de clientes y como hecho diferencial frente a la competencia y así asegurarse mayor cuota de mercado.

Sin embargo, las relaciones que definen la meta-gobernanza (la forma en que se gobiernan las relaciones entre Estado, mercado y sociedad civil -jerárquica, por redes, de mercado, en combinación...-), son muy distintas en cada contexto específico obedeciendo a razones históricas, políticas, culturales, religiosas, etc. (Jessop, 2004; Sorensen \& Torfing, 2009; Swyngedouw, 2005). Donde la sociedad civil (en esencia de donde emergen tanto la gobernación como el mercado) no es lo suficientemente vigorosa, esta acaba por ser sometida (como la parte paria) a los intereses de la clase dirigente o a la de los poderes económicos que, como no resulta difícil de comprobar (tal y como demuestra la actual situación de crisis y de pérdida progresiva de derechos sociales asociada a ella), muchas veces supeditan al propio Estado. No puede hablarse entonces de democracia plena o democracia real (Romero \& Farinós, 2011). Cómo poder revertir esta situación pasa necesariamente por empoderar a la sociedad civil, así como por una reinterpretación de su función. Gobierno 
y gobernanza, siendo entonces distintos, más que antagónicos resultan complementarios; si no consecutivos en forma de espiral ascendente (Farinós, 2008, p. 14).

Este nuevo buen gobierno, sin embargo, no asegurará la gobernabilidad. El concepto de gobernabilidad pone en relación actores y reglas, sometiéndose todos ellos, independientemente de su capacidad de poder sabotearlas, a las mismas. Se refiere, por tanto, al grado de prevalencia de las mismas y por el nivel de conflicto que puede ser gestionado o resuelto siguiendo las reglas y procedimientos establecidos o acordados en vigencia. Seguimos por tanto la interpretación de gobernabilidad de Prats (2005a, p. 28): "atributo de las sociedades que se han estructurado sociopolíticamente de modo tal que todos los actores estratégicos se interrelacionan para tomar decisiones de autoridad y resolver sus conflictos conforme a un sistema de reglas y procedimientos formales e informales... dentro del cual formulan sus expectativas y estrategias". Con todo, determinados actores hegemónicos, aunque existan unas reglas de funcionamiento común (garantía de un Estado de derecho), pretenden escapar de ellas en función de sus propios intereses. Es justamente aquí donde se centra la actividad y el control de la sociedad civil en la custodia del interés general y del bien común.
Esta es, precisamente, la razón de fondo que ayuda a explicar la mayor importancia concedida a la participación como elemento clave y principal, que no único, de la gobernanza. Es evidente que viejos modelos y fórmulas reglamentistas, burocráticas y dirigistas no han dado los resultados esperados; pero tampoco la desregulación y la fe ciega en los mercados. La posible solución apunta en varias direcciones: procurar nuevos enfoques, métodos y rutinas para producir y aplicar las políticas; facilitar los mecanismos de control y dación de cuentas (monitoreo y evaluación de las políticas públicas); lograr el desarrollo y mantenimiento de una adecuada cultura política y territorial, a la que justamente pretenden contribuir las dos anteriores. Un amplio objetivo a largo plazo para el que la mejora de las relaciones y una más efectiva colaboración entre la academia, los técnicos y tomadores de decisiones, los y la sociedad civil, se erigen como elemento facilitador.

En relación con el debate central sobre la relación Estado-ciudadano, se comparte el argumento de Joan Prats sobre lo que debe entenderse por modelos de gobernanza en cada caso y en cada contexto específico, así como sobre el papel central de la esfera pública (enfoque neoinstitucionalista de una gobernanza reforzada): "No creemos en la gobernanza como sustituto del gobierno, sino en la go- 
bernanza con gobierno, como modalidad de gobernación. La gobernanza no puede diluir, sino fortalecer y legitimar la autoridad democrática" (Prats, 2005b, p. 171). Pero llegados a estas alturas cabe reinterpretar su función, regresando a la causa que le da origen y a su objetivo de velar por la y el interés general, la de Aristóteles. Ante el riesgo de convertirse en el mar menor ("el menos malo de los sistemas"), hay que volver a los fundamentos de la propia democracia: la ciudadanía y su 'deconstruible' capacidad de organización para la defensa de valores e intereses individuales y colectivos (la cívica).

La mayor dificultad para ello hace tiempo que se encuentra en el ámbito de la política, primordialmente en el ámbito de los partidos políticos. Recuperar la cultura política del acuerdo se presenta condición necesaria. La gobernanza democrática descansa, básicamente, sobre los principios de coordinación y cooperación. En consecuencia, sus progresos quedan dificultados en la medida en que no exista voluntad de acuerdo entre los actores políticos y entre estos y los actores económicos y sociales. Esta circunstancia se ve agravada por el hecho de que la provocada escasa densidad del tejido social favorece que la 'política oficial' (especialmente los partidos dentro de la gobernación) ocupe la mayor parte del espacio.
Por ello cabe concluir que la gobernanza territorial, para poder serlo, debe ser gobernanza democrática, como diría Pascual (2011); es decir, debe involucrar toda la constelación de actores y no únicamente intereses particulares. Las autoridades públicas, a cualquiera de los niveles, lógicamente todavía juegan un papel central. Los gobiernos nacionales y regionales deben reforzar su papel en el establecimiento del marco, esto es, de las precondiciones para que puedan producirse las acciones y procesos de gobernanza territorial. A ellos les corresponde legislar y en la solución de conflictos, a lo que pueden contribuir ampliamente a través del establecimiento de un adecuado marco regulador y de gestión de la información, pudiendo llegar a convertirse así, a través del buen ejemplo de su propia gestión, en un buen referente de apertura y participación.

\section{La necesidad de una adecuada cultura política... la piedra filosofal}

Los cambios territoriales son cada vez más dinámicos y apuntan en diferentes direcciones, lo que obliga a pensar en nuevos conceptos, procedimientos e instrumentos capaces de adaptarse a las situaciones cambiantes. La ontología del territorio, la experiencia y el entendimiento que de él tenemos, así como las formas 
de gobernarlo que de ello se derivan, dependen de las propias narrativas o e, históricamente específicas, aceptadas por las comunidades que lo habitan, y articuladas de forma que permitan interpretar de forma simple $\mathrm{y}$ efectiva las relaciones y procesos complejos propios de cada territorio, con el objeto de poder actuar sobre ellos en la práctica.

Si en la planificación estratégica de primera generación lo importante eran los proyectos y su impulso, en la de segunda lo principal es la articulación del proceso que organiza y estructura las relaciones y las interacciones sociales. Estas dependerán de la capacidad de organización (capital social), pero también de la capacidad de discusión y decisión (innovación social); en función, a su vez, del capital intelectual disponible y, por ende, de la racionalidad discursiva posible. Las sociedades más complejas y diversas requieren de valores y actitudes compartidos derivados de la propia historia y cultura que favorezcan la interacción y la densidad de relaciones que promuevan la necesaria innovación socio-territorial que acabe por dar lugar a nuevas "prácticas transformadoras". Como señala Albrechts (2009), para hacerlas posibles se requiere de un riguroso $\mathrm{y}$ crítico análisis de los factores técni$\cos$, legales, financieros, organizacionales y de propiedad que afectan las posibilidades de realización concreta de los planes y proyectos estratégicos, para así poder investigar creativamente sobre los instrumentos, herramientas, medios y rutinas más apropiados.

Una buena planificación del desarrollo territorial sostenible clama, además de por la participación, por el "regreso de la política". Uno de los parámetros cruciales para un adecuado progreso de las nuevas formas de gobernanza territorial en cualquier sociedad es la madurez del sistema político y la cultura política existente. Contrariamente no son pocos los casos de sociedades escasamente informadas y altamente dependientes de las relaciones clientelares (Farinós \& Romero, 2008, p. 8-9; Wassenhoven, 2008). Como escribía Madoery (2003), los retos que impone un nuevo patrón de desarrollo territorial sostenible precisa de un "consenso social y sectorial respecto de las políticas a implementar, en un intercambio creciente con los diferentes actores representativos y un criterio racional y estratégico en la utilización de los recursos". Estado, mercado y sociedad civil no pueden responder a estos desafíos solos o por separado, pero sí mediante la conjunción de esfuerzos entre ellos.

En el actual contexto económico, social y político de honda crisis por la que atraviesan no pocos Estados nacionales, especialmente europeos, no son pocas las reacciones y los esfuerzos por tratar de plantear y brindar 
alternativas de solución. La actual situación en la que nos encontramos inmersos empieza a dar muestras suficientes de una violencia estructural con efectos sobre los ciudadanos que se ven sometidos a una angustiosa, progresiva y acelerada reversión de sus derechos; escudada en supuestos argumentos indiscutibles (en realidad falacias muchos de ellos) y en el manido recurso a la táctica de los hechos consumados y a la supremacía de lo posible o factible sobre lo necesario.

Frente al fatalismo, entendido como incapacidad para dirigir el propio futuro, y la falta de esperanza sobre cómo poder vislumbrar la luz al final del túnel sin tener que renunciar a los derechos adquiridos, la actuación de la ciudadanía, organizada en formas diversas (de entre las que la democracia ha resultado ser la opción predilecta -pese a sus deficiencias, o tal vez por ellas-). Las posibilidades y nivel de participación de los ciudadanos dependen tanto del contexto político (y la forma en la que se apoya o desincentiva dicha participación -directa o indirectamente-) como de la propia cultura política.

La idea del demos al estilo ateniense o afrancesado -indiferenciación del sujeto en tanto que forma parte de una misma comunidad de intereses con su propia narrativa, entendida como el conjunto de significados, discursos, prácticas compartidas y formas de entender los problemas y la forma de darles solución (Geertz, 1973) - puede parecer atractiva. Sin embargo, pueden existir varias culturas políticas o comunidades de intereses dentro de una misma población (Vallés, 2004, p. 256). Almond y Verba (1970) identificaban tres tipos ideales de cultura política: 'cívica o participativa', cultura 'del súbdito' y cultura 'localista o clientelar'. En la cultura cívica o participativa los ciudadanos aceptan el juego de representación política y están dispuestos a incorporar sus demandas (inputs) en la acción de gobierno mediante una participación activa. En este caso la distancia entre administración y administrados es estrecha, su comunicación fácil, y se practica una mutua entre electos y electores. En la cultura del súbdito los individuos se muestran atentos a las decisiones de las instituciones que afectan sus vidas, siendo poco conscientes de su capacidad de influencia. Actúan más como espectadores que como protagonistas. Esperan más el 'output' de la acción de gobierno que en suministrar el 'input' para que esta se dirija en la dirección deseada. Predomina en este caso el fatalismo frente a la capacidad de liderar el propio futuro. Finalmente, en la cultura localista o clientelar los individuos (cabría decir tanto ciudadanos de a pie como tomadores de decisiones) se encuentran poco interesados por el sistema, salvo si se puede manejar a conveniencia, 
por ello prestan especial atención a las relaciones personales inmediatas que puedan reportarles los beneficios esperados.

La participación de la ciudadanía, sujetos con derechos, entre ellos el de poder decidir su propio futuro, genera las condiciones necesarias para un adecuado desarrollo de la comunidad y el espacio o territorio que habita. Sin embargo, el contexto institucional o de gobierno puede debilitar, en lugar de nutrir, dicha participación. Es en ese momento cuando la revisión de la acción de gobierno y de la misma forma en que se gestiona la democracia debe entrar a revisión. No cabe otra opción ante el uso torticero e interesado que desde el poder se hace de la gobernación, de las instituciones de gobierno y de las reglas del juego democrático, para empezar a exigir unas nuevas políticas en clave más deliberativa (Habermas, 2003). Es el ultimátum ante el que se reacciona en forma de movimientos ciudadanos como los vividos en las distintas "primaveras".

Fomentar una adecuada gobernabilidad pasa por hacer más efectivas las instituciones y las normas, la transparencia (en oposición a la opacidad), la participación, la capacidad de control y respuesta, la dación de cuentas (en lugar de la impunidad) y la primacía de la ley (igual para todos, en lugar de diferenciar entre poderosos, políticos y el resto de la gente). Sin esta -en terminología de Aristóteles- el buen gobierno y la democracia no son posibles. Tampoco con la manifiesta tendencia actual a la concentración o mezcla de poderes (legislativo, ejecutivo y judicial), frente a la separación propugnada desde el siglo XVIII en Estados Unidos y Francia. Desde el otro lado, el culto al individualismo, la indiferencia y la apatía de la comunidad han alimentado tanto el desinterés por la política y por su control, abriendo mayores posibilidades para el uso inadecuado sino directamente el abuso de las instituciones democráticas y, finalmente, a una pérdida de confianza en el sistema político.

Pero iniciar el cambio que abra las puertas a nuevas prácticas de gobernanza territorial, por lo visto hasta ahora, no resulta tarea fácil. Entraña la recuperación o modificación (a veces incluso la creación) de valores, creencias y rutinas, individuales, grupales e instrumentales. Además, existen algunos condicionantes que afectan a los sistemas de planificación implantados y a su posible cambio: factores estructurales, políticos, culturales y profesionales; las propias actitudes ante la ordenación del territorio; y la voluntad política por parte de las instituciones involucradas. Se trata de poder avanzar desde la innovación social y socioinstitucional a la socioterritorial, con nuevos actores 
en la arena política, lo que presupone un cambio de pesos específicos y de responsabilidades, más compartidas y negociadas, a la hora de promover nuevas estrategias y políticas de desarrollo de los territorios, que ahora se entiende no pueden basarse sino en el adecuado aprovechamiento de las especificidades y condiciones locales (desde dentro y para los de dentro, fundamentalmente). Es entonces cuando se unen la buena gobernanza, la condición de gobernabilidad de los territorios y la meta del desarrollo territorial justo, digno y sostenible.

El cambio real requiere una dosis adecuada de inteligencia territorial, tiempo y dedicación; es decir, un compromiso y cultura cívica para la toma decisiones sobre las cuestiones importantes que afectan a la comunidad. Para ello la información y el conocimiento sobre los procesos territoriales resultan pieza clave, como se discute a continuación.

\section{La necesidad de información e inteligencia territorial... y gubernamental}

Como decíamos, la puesta en práctica de la gobernanza territorial requiere el desarrollo de una nueva cultura territorial. Esta se sustenta en el desarrollo de una suficiente inteligencia territorial, una cuestión que se relaciona tanto con el desarrollo de un suficiente capital intelectual sobre el territorio, como de información e indicadores territoriales con los que poder identificar las dinámicas territoriales existentes y evaluar el impacto de las políticas. Por capital intelectual entendemos en este caso el nivel de conocimiento disponible para los actores implicados en el desarrollo territorial (conocimiento del sistema territorial y de cómo evoluciona, de los procesos socioeconómicos y su impacto sobre el desarrollo territorial, y de las formas en las que operan las instituciones). Gracias a él es posible desarrollar un entendimiento compartido y así dotarse de un marco común de referencia que permita la cooperación entre actores. Resulta elemento fundamental para poder definir de forma común un problema y acordar una estrategia y una visión para el territorio de cara al futuro.

El capital intelectual aparece, de este modo, como una precondición, pero también como un recurso. La necesidad de una información territorial suficiente sobre la que poder basar la toma de decisiones y los consiguientes procedimientos de evaluación, mejorando así la calidad de la planificación y los resultados de las actuaciones, nos traslada a su vez al tema de los observatorios territoriales, que pueden contribuir decididamente al conocimiento de la realidad territorial (Farinós, 2007, 2011b). En los últimos años se ha venido expe- 
rimentado una creciente proliferación de observatorios, relativos a diferentes escalas o ámbitos de actuación, generalmente de carácter sectorial en lo temático, más interesados por cuestiones concretas para las que se trata de recopilar indicadores más o menos oficiales, en lugar de definir indicadores consensuados y comparables entre distintos ámbitos de la misma escala o entre escalas y administraciones distintas (de la local a la supranacional). Esta multiescalaridad resulta clave de cara al futuro, ya que, en última instancia, haría posible acordar estrategias de cohesión territorial a distintos niveles. Por lo general no se va mucho más allá de la mera recopilación de datos estadísticos disponibles, o del enlace a fuentes de información e indicadores relacionados ya elaborados. Menos habitual resulta dar un paso más para proponer indicadores propios con los que poder evaluar las alternativas de actuación política desarrolladas sobre el territorio en cuestión.

Esta información no solo resulta de utilidad para los tomadores de decisiones, sino también para otros actores del sistema, de forma individual $u$ organizada (emprendedores económi$\cos$ y sociales), que pueden servirse de ella para poder participar (orientar) en el diseño, ejecución y evaluación de las políticas (acciones), de las que finalmente debieran responder los tomadores de decisiones públicos en aras de una necesaria y deseada transparencia y dación de cuentas. De esta forma todos los ciudadanos pueden participar y contribuir a la definición de sus propias opciones de futuro, no solo como meros usuarios de la información, sino que incluso pueden llegar a convertirse en generadores o actualizadores de la misma (al estilo de las wiki o Web 2.0)

La medición del desarrollo de la sociedad es una actividad que cada vez se encuentra más presente en los ámbitos políticos y científicos. Podría establecerse un continuo sobre el que situar las cuatro posibles funciones de estos observatorios (Bovar \& Perony, 2006, p. 25). De menor a mayor nivel de exigencia: observación (recogida de datos y elaboración de indicadores), estudio (procesado de la información disponible para la elaboración de análisis sobre situación y procesos, combinando análisis y métodos cuantitativos y cualitativos), prospectiva (establece, sobre la fase de diagnóstico, unas prognosis -a corto/medio- y unos escenarios de futuro -a largo-) y evaluación (los indicadores son establecidos, en el mismo proceso de diseño de políticas, planes y programas, para hacer un seguimiento de los mismos y comprobar hasta qué punto se cumplen los objetivos propuestos). De forma genérica pueden agruparse estas cuatro funciones en dos: de señalización (observación y estudio) y de evaluación (De Vries, 2009). 
La información territorial permite la evaluación del impacto de las políticas y los sistemas de planificación existentes (Farinós, 2005), la visualización de futuras tendencias, la elaboración de escenarios y la propuesta de nuevos conceptos y soluciones territoriales. El resultado deseado es poder conseguir un buen soporte para la toma de decisiones y la planificación del desarrollo territorial, asumiendo el reto de integrar la dimensión territorial en la planificación del desarrollo.

Pero la realidad sigue mostrándose bien distinta. A pesar de ello, o tal vez por ello, es importante reconocer que más de lo mismo no será suficiente. Es la razón que anima la discusión, apuntada en los apartados iniciales de este texto, sobre las relaciones entre gobierno, buen gobierno, nuevo gobierno, e-gobierno, gobernanza o gobernabilidad; todos ellos relacionados como parte de un mismo fin, pero distintos entre sí. De fondo el debate sobre si el binomio ciudadano-Estado es posible y cómo, tal y como apuntábamos párrafos más arriba; sobre si el equilibrio y sinergias positivas entre gobierno-poder-democracia real es posible; de si se respeta el contrato social entre gobernantes y ciudadanos (y si existen mecanismos de corrección en el caso de no cumplirse con el interés o los intereses generales y con el programa de gobierno anunciado que vincula a gobernantes y gobernados -incluso si este es explícito y se conoce o no-); de si existe una verdadera separación de poderes (no ya solo de los tradicionales legislativo, ejecutivo y judicialsino de estos con el llamado cuarto poder -la prensa-, en unos momentos en los que los grandes medios de comunicación pasan a ser un actor clave del juego político que, lejos de constituirse en la conciencia o la 'garganta profunda' del atasco político incluso se vanagloria de poner y quitar presidentes y gobiernos); de si una administración más eficiente es condición suficiente, e incluso hasta necesaria, dadas las críticas a la burocracia y las propuestas de solución propuestas: nuevas gerencias públicas y deseos de un Estado mínimo, en la senda del pensamiento único y de la concepción del ciudadano como cliente, de la regresión del Estado social y de derecho.

Frente a ello, la reivindicación de una democracia activa y deliberativa, que trata de tender nuevos puentes entre ciudadanía-expertos-técnicostomadores de decisiones; una cuestión no exenta de problemas, tanto por la propia complejidad y carácter no cíclico -unívoco- del proceso de toma de decisiones, como por reticencias y desconfianzas persistentes. A esta nueva democracia real contribuye una mayor información sobre la que basar el conocimiento y la inteligencia (comprensión del problema y 
de las posibles alternativas de solución), a partir de las que poder crear opinión y finalmente la decisión de actuar. Las nuevas formas de planificar el desarrollo territorial, con un carácter más participado y estratégico, ayudan. Del modelo de planificación territorial comprehensivo y del plan director (de racionalidad perfecta, infalible, donde el técnico o académico tenía la última palabra), del despotismo ilustrado de las élites profesionales y políticas (que ha dado como resultado la emergencia no solo de no pocos efectos no deseados -impactos- sino reacciones de la población en forma de conflictos territoriales) se transita hacia una nueva planificación colaborativa de estilo discursivo, apoyado sobre la información y el capital territorial. Una nueva planificación territorial donde las relaciones entre certezas (de los planes directores o 'master plan' a las planificaciones flexibles), seguridades (compromisos entre niveles y actores para asegurar que lo planificado se cumple) y el nivel de concreción (del plan al proyecto y su coordinación) cambian. Una planificación basada en evidencias y en los deseos de la población concernida; una nueva planificación estratégica de segunda generación, de base democrática y participada, de la que la tarea de evaluación forma parte inseparable. A ella se dedica el último apartado que sigue.

\section{5. (R)evolución en la evaluación de las políticas públicas. ¿Por qué evaluación territorial?}

Los cambios territoriales son cada vez más dinámicos y apuntan en diferentes direcciones, lo que obliga a pensar en conceptos, procedimientos e instrumentos capaces de adaptarse a las situaciones cambiantes. El papel de la geografía resulta clave en este sentido, mediante el desarrollo de unas metodologías claras, rigurosas y contrastables. No solo para analizar los procesos y la funcionalidad del territorio, sino también para la evaluación del impacto de las políticas y los sistemas de planificación existentes, para visualizar futuras tendencias y proponer nuevos conceptos y soluciones territoriales. Los geógrafos son uno de los colectivos llamados a contribuir en el desarrollo de una nueva cultura del territorio, combinando sentido de pertenencia y acción.

La evaluación de políticas está recibiendo cada vez más atención gracias a una mayor demanda social y presión mediática, de las organizaciones no gubernamentales y las instancias supranacionales. La determinación y seguimiento de los efectos de la política, particularmente los ambientales y más recientemente sociales, comienza a ser exigida, no solo por los acuerdos internacionales en materia 
de desarrollo sostenible ${ }^{5}$, sino también por las exigencias de transparencia en el marco de la globalización y liberalización comercial y financiera.

La evaluación de políticas se ve entonces como una práctica basada en una información pertinente, que permite informar de manera objetiva (basada en evidencias) de los efectos de las acciones públicas. Esta actividad de evaluación es todavía más importante cuando instituciones y gobiernos contemporáneos tienden a fundamentar su legitimidad no solo institucionalmente sino también por sus resultados. La actividad evaluadora permite al Estado y a los ciudadanos disponer de una mayor información acerca de las consecuencias de sus decisiones y acciones $^{6}$.

Si hasta la fecha la evaluación para valorar la aplicación, rentabilidad y resultados de medidas gubernamentales ha sido la predominante, algo ha cambiado recientemente. Las intervenciones del Estado moderno son más amplias, su situación más complicada y sus consecuencias potenciales de largo alcance, por ello se considera conveniente involucrar la ciencia y la investigación social para dirigir sus

5 Como por ejemplo el compromiso de desarrollar la Agenda 21 Local, obtenido en la Cumbre de Río de Janeiro de 1992, el Convenio Europeo del Paisaje de 2000, el Protocolo de Kyoto en la lucha contra los efectos contra el Cambio Climático, el cumplimiento de los Objetivos del Milenio, cuya revisión se encuentra prevista en $2015 \ldots$ entre otros.

6 De nuevo nos acercamos a la idea de nueva planificación territorial estratégica, democrática 0 de segunda generación (Albrechts, 2010; Pascual, 2007, 2011). operaciones y prever los impactos. El objetivo es llevar a cabo una reflexión que ayude a orientar mejor el futuro y permita a los tomadores de decisiones actuar de la forma más eficiente posible. Cuestión distinta es la de cómo llegar a establecer de forma efectiva dicha colaboración, implicando también a la propia ciudadanía en un ejercicio de transparencia y corresponsabilidad propia de una nueva forma de gobernanza (democrática) que haga viable la gobernabilidad de los territorios. A ello puede contribuir en gran medida esta dación de cuentas y evaluación de propuestas e impactos. A través de una adecuada evaluación de impacto territorial (EIT), una forma más (ligada a la observación territorial) de manifestación de inteligencia territorial.

En materia medioambiental se empezó hablar de evaluación ambiental en 1969, cuando Estados Unidos promueve la Ley Nacional de Política Ambiental, estableciendo los requisitos y procedimientos para la misma. Sin embargo no llega a diferenciar entre la evaluación de proyectos y la de políticas, planes y programas, aunque la práctica evidenció una mayor aplicación en proyectos. Ya en la década de los 80, en el marco del IV, V y VI Programa de Medio Ambiente de la Unión Europea, se promueve y se establece la EIA para planes y programas. Se utiliza como herramienta preventiva de la gestión ambiental en la tarea de planificación, logrando enfatizar la importancia de la integración de los objetivos ambientales en los primeros estadios de la planificación. Habrá que 
esperar hasta 2001 para que la evaluación ambiental estratégica (EAE) de planes y programas se incorpore al ordenamiento jurídico de la Unión Europea, con desarrollos posteriores ${ }^{7}$. Sin embargo, la normativa comunitaria en materia medioambiental no genera derecho ni competencia alguna en materia de planificación territorial en el marco de la UE (Parejo, 2007). Tampoco la EAE ha dejado de adolecer de algunos problemas motivados por un entendimiento de la misma más cercano a una evaluación de impacto ambiental (EIA) de planes y programas que a una verdadera evaluación ambiental y de carácter más estratégico que la pueden acercar a la EIT (Farinós, 2011a). También obedece a un enfoque más sectorial que integral o comprehensivo, olvidando la incorporación de las cuestiones territoriales, de las que deberían haber sido absolutamente indisociables (Eggenberger \& Partidàrio, 2000).

\footnotetext{
7 Se trata de la Directiva 2001/42/CE del Parlamento Europeo y del Consejo de 27 de junio de 2001 relativa a la evaluación de los efectos de determinados planes y programas en el medio ambiente. (DOCE n L 197/30, 21-07-01), cuyo objetivo es la integración de todas las consideraciones medioambientales en el proceso de preparación y adopción de planes y programas al objeto de poder promover un desarrollo más sostenible.

Directiva 2003/35/CE del Parlamento Europeo y del Consejo de 26 de mayo de 2003, por la que se establecen medidas para la participación del público en la elaboración de determinados planes y programas relacionados con el medio ambiente y por la que se modifican, en lo que se refiere a la participación del público y el acceso a la justicia, las Directivas $85 / 337 / C E$ y 96/61/CE del Consejo. (DOCE $\left.n^{0} \mathrm{~L} 156 / 17,25-06-03\right)$.

Directiva 2011/92/UE del Parlamento Europeo y del Consejo, de 13 de diciembre de 2011, relativa a la evaluación de las repercusiones de determinados proyectos públicos y privados sobre el medio ambiente. (DOUE L 26 /1 de 28.01.2012)
}

De esta forma, hoy en día, coexisten tres evaluaciones: la EIA, la EAE y la EIT, con unos pesos específicos bien distintos. Es el enfoque territorial el que menos presencia tiene, excepto cuando la planificación es explícitamente territorial, como en el caso de la ordenación del territorio. Si la EAE privilegia los aspectos ambientales, la EIT se preocupa de aspectos económicos, sociales y ambientales, a través de la idea de cohesión territorial. Dado que la EIT ha llegado más tarde, la cuestión es llegar a definir el, o los objetivos y la, o las metodologías de la EIT, y su relación (¿qué aporta de nuevo?) con otras evaluaciones como la EAE o la paisajística, más reciente.

Para los planificadores europeos la EIT parece querer orientarse a una evaluación integral del objetivo de la cohesión territorial, lo que lleva a considerar a esta como una 'New Spatial Strategic Planning' (Albrechts, 2010) a escala europea, como crisol de las políticas con impacto territorial, que deben ser coordinadas mediante una previsión de sus impactos potenciales ${ }^{8}$. No es seguro que fuera esto lo que pudo estar en mente en el proceso de elaboración del documento de la Estrategia Territorial Europea -ETE- (CE, 1999), que incluye alguna referencia de naturaleza más concreta a la necesidad de evaluar los impactos territoriales de las políticas sectoriales (en especial la de infraestructuras), a fin de poder

\footnotetext{
8 Sobre esta misma cuestión, la de la relación entre planificación territorial, evaluación y política europea, escribían ya a finales de los años 1990 Dabinet y Richardson (1999).
} 
coordinar sus efectos territoriales (horizontalmente) y trabar mejor los diferentes niveles de gobernanza en la UE (verticalmente). Pero lo cierto es que esto ha acabado siendo así gracias a los avances de la idea de cohesión territorial, especialmente a partir de 2005 hasta su inclusión en el nuevo derecho primario de la UE resultante de la reunión del Consejo Europeo de Lisboa en diciembre de 2007. El programa de acción de la Agenda Territorial Europea que seguía a la reunión de Leipzig de 2007 consideraba la EIT como uno de sus ejes prioritarios de trabajo, junto a las relaciones urbano-rurales y a una mejor coordinación entre la planificación urbana y territorial.

La mayor consideración que ha tenido la ordenación del territorio a nivel europeo (a través del documento de la ETE, del proceso de la Agenda Territorial Europea y de la idea, principio y ¿política? de cohesión territorial) se ha visto acompañada por una mayor importancia concedida a la evaluación, tanto en el proceso de elaboración de políticas, planes y programas, como en el de implementación y gestión (Valenzuela \& Soria, 2011). A la vez, ha sido un factor de presión importante a la hora de integrar los factores territoriales en la evaluación, a lo que también han contribuido los rápidos e importantes cambios del uso del suelo, muchas veces con claros efectos negativos desde el punto de vista ambiental.

La complejidad de la EIT reside justamente en su amplitud, dado que la ordenación del territorio es un campo in- tersectorial y transdisciplinar. La EIT buscaría así determinar una amplia gama de impactos que pueden causar tanto las políticas como los grandes proyectos desde una visión multidimensional, contemplando las complejas relaciones entre las dimensiones económica, social y medioambiental presentes en un mismo territorio. Ante la complejidad de tal reto (para un mayor detalle vid. Farinós, 2011a), pero también ante las exigencias de mayor simplicidad, no más procedimientos, ni burocracia ni costes en el proceso de planificación, las miras se rebajan, de dos formas:

a) Integrando la dimensión territorial en la EAE (por cuanto las cuestiones medioambientales encuentran perfecto acomodo en el análisis del medio físico) fundamentalmente en la fase de 'scoping' y en el Informe de Sostenibilidad Ambiental (ISA), considerando el modelo territorial escogido como umbral o referencia sobre el que poder testar la sostenibilidad del plan (Gómez, Gómez \& Gómez, 2011; Pereira, 2011; Serrano, 2011), contribuyendo de esta forma a dotar de un estatuto propio y diferenciado a la EAE respecto de la EIA.

b)Centrándose en la evaluación del grado de cumplimiento de objetivos de planes y proyectos de ordenación territorial (Faludi, 2011), y en los efectos y cambios que las actuaciones provocan en el sistema territorial. No es poco; presupone la existencia de plan para el territorio, 
presupone también que se cuenta con una inteligencia territorial (información, sistemas de indicadores y método de evaluación -concretados en forma de observatorios... o no-) y que también se cuenta con voluntad política. No se trata, por tanto, de una simple tarea de seguimiento, monitoreo o control. En la construcción de estos sistemas de seguimiento y evaluación territorial deben tenerse en cuenta tres cuestiones fundamentales (Jaeck, 2009, Marques, 2011): ¿qué monitorear o evaluar? (planes y proyectos territoriales); ¿cómo hacerlo? (mediante evaluación continua por medio de una serie de indicadores definidos en el mismo proceso de elaboración de los planes de ordenación del territorio); ¿para quién? (en defensa de la calidad de vida de la población y del interés general).

Sea cual sea la opción final escogida, cualquiera de ellas supone completar la actividad de evaluación tratando de cambios, tendencias e impactos territoriales en sentido amplio, o sobre el medio físico más específicamente; comprobar si la evolución territorial está en sintonía con el plan o estrategia territorial decidida; determinar un conjunto de indicadores (al menos un juego de indicadores específicos -útiles, relevantes, efectivos, eficientes y durables o fácilmente actualizables-) que permitan evaluar los elementos o variables críticos o estructurales seleccionados. La base para una nueva situación de suma mayor que cero (win-win) tanto para ciudadanos como para los tomadores de decisiones y los políticos, para los técnicos y académicos involucrados en los procesos de planificación, y para el mismo territorio, que gana en calidad y capacidad de atracción y rendimiento. Tratemos de hacerlo fácil sin perder perspectiva ni rigor.

\section{A modo de conclusión}

La respuesta al problema de la desigualdad social y territorial, problemas hasta ahora contemplados de forma disyunta y con un claro orden de prelación (antes que nada social), conviene ser planteada en estos momentos de una forma combinada, como el mejor modo de poder afrontar de forma resiliente los nuevos retos del siglo XXI.

Las acciones de gobernanza territorial deben territorializarse, ser puestos en relación con el capital territorial existente. Ello demanda una revalorización del territorio y la mejora de una nueva cultura territorial común, para cuya consecución el papel de los actores públicos es crucial. En este sentido conviene prestar mayor atención a la gestión y ordenación territorial, especialmente a la planificación estratégica, en tanto representa una muy buena opción para mejorar la coherencia, y efectividad, de las políticas. Coordinación y cooperación constituyen elementos básicos de la buena gobernanza territorial. Junto a una renovada ética pública, la nueva gobernanza territorial se asienta sobre los valores democráticos y de participación ciuda- 
dana que hagan posible la deseada gobernabilidad efectiva de los territorios.

La planificación del desarrollo territorial puede actuar como guía del proceso político de toma de decisiones. Las estrategias a partir de las cuales se instrumenta la política territorial son diversas. El resultado ha sido la fragmentación entre las distintas políticas sectoriales y la descoordinación entre los niveles de poder involucrados. Una mejor y más eficiente coordinación permitiría otorgar un nuevo espacio de acción, hoy baldío, a una nueva ordenación territorial reconstituida, como "política crisol" de otras políticas, con el objetivo de un nuevo desarrollo en dignidad y de la cohesión territorial. Por ello resultará un campo especialmente indicado para el desarrollo de nuevas prácticas de gobernanza. Bajo estas nuevas premisas el espacio se produce, se territorializa, en forma de nuevas estrategias o visiones territoriales, a partir de las expectativas, deseos e intereses de la ciudanía y los actores locales.

Siguiendo el enfoque de la complejidad relacional, los procesos de planificación territorial estratégica requieren saber detectar y aprovechar las múltiples dinámicas existentes en un lugar, identificando eficientemente los factores clave para ponerlos a disposición de la acción colectiva de acuerdo con las propias cualidades de la localidad. Esto presupone la recuperación tanto de la idea de síntesis geográfica como de la individualidad (unicidad) de la región o espacio geográfico. Todo esto resulta más fácil a escala local, en la que el territorio, los actores y las instituciones se reconocen más fácilmente y con los que la población se identifica mejor.

Ello nos habla de la conveniencia tanto de un nuevo estilo de gobierno del territorio como de planificación, con una mayor presencia de la evaluación y una mejor interacción y observación entre las distintas administraciones y departamentos responsables de las políticas públicas. También de una nueva cultura política, en la que el control mutuo entre ciudadanía, élites y políticos sea lo habitual. Es en las sociedades diversas y plurales donde más se requiere del diálogo, en sentido deliberativo, a fin de lograr consensos, acuerdos y contratos entre los distintos niveles político-administrativos (gobernanza multinivel). Pero no sin antes haberlos acordado entre la ciudadanía y cada uno de estos niveles de poder. Por ejemplo mediante la formulación de estrategias locales basadas en las características propias de cada territorio, de acuerdo con las voluntades y compromisos de sus habitantes.

Se trata tanto de promover una nueva cultura territorial y urbana en la sociedad civil como, especialmente, de actuar sobre los futuros profesionales para poder prepararlos, además de en las técnicas, en los procesos, rutinas y estilos de trabajo (una especie de código deontológico). No solo de actualizar su capacitación para que puedan seguir participando (en mejores condiciones competitivas) en la elaboración 
de los planes, sino también para que puedan desarrollar nuevas funciones de mediación y empoderamiento de la población en los planes de participación pública que contempla la actual legislación y responde bien a los principios de una nueva planificación colaborativa o deliberativa. Ello permitiría situarnos a los geógrafos como colectivo en una ventajosa situación de facilitadores para los tomadores de decisiones, en un nuevo estilo tanto de gobierno del territorio como de planificación, con una mayor presencia de la evaluación y una mejor interacción y observación entre las distintas administraciones y departamentos responsables de las políticas públicas cuyo impacto territorial se prevé y evalúa.

\section{Referencias}

Albrechts, L. (2004). Strategic (spatial) planning reexamined. Environment and Planning B: Planning and Design, (31), 743-758.

Albrechts, L. (2006). Shifts in strategic spatial planning? Some evidence from Europe and Australia. Environment and Planning A, 38(6), 1149-1170.

Albrechts, L. (2009). Bridging the Gap: From Spatial Planning to Strategic Projects. En Farinós, J.; Romero, J. y Salom, J. (eds.), Cohesión e inteligencia territorial. (pp. 85-104). Valencia: PUV / IIDL, Colección Desarrollo Territorial no 7.

Albrechts, L. (2010). More of the same is not enough! How could strategic spatial planning be instrumental in dealing with the challenges ahead? Environment and Planning B: Planning and Design, (37), 1115-1127.

Albrechts, L., Healey, P. \& Kunzmann, K. (2003). Strategic Spatial Planning and Regional Governance in Europe. Journal of the American Planning Association, (69), 113-129.

Almond, G. \& Verba, S. (1970). Cultura cívica. Boston, MA: Little, Brown and Company.

Bovar, O. \& Peyrony, J. (2006). Le cas français de l'Observatoire des territoires. L'évidence par la prospective ou par l'observation? Disp., 165(2), 25-33.

Buroway, M. (2005). 2004 Presidential Address: For Public Sociology. American Sociological Review, (70), 4-28. Recuperado de: (http://burawoy.berkeley.edu/PS/ASA\%20 Presidential\%20Address.pdf

Comisión Europea -CE- (1999). ETE. Estrategia Territorial Europea. Hacia un desarrollo equilibrado y sostenible de la UE. Luxemburgo: Oficina de Publicaciones Oficiales de las Comunidades Europeas.

Chilvers, J. \& Evans, J. (2009). Understanding Networks at the Science-Policy Interface. Geoforum, (40), 355-362.

Dabinet, G. \& Richardson, T. (1999). The European spatial approach: the role of power and knowledge in strategic planning and policy evaluation. Evaluation, 5(2), 220-236. 
Davoudi, S., Farinós, J., Paül, V. \& De Vries, A. (2009). Desarrollo territorial sostenible a partir de la cohesión territorial: entre el crecimiento económico, la cohesión social y la sostenibilidad medioambiental. En J.M. Feria (ed.) Territorios, sociedades y políticas (pp. 199-238). Sevilla, España: UPO-AGE.

De Vries, A. (2009). Gestión de información territorial para la toma de decisiones. En J. Farinós, J. Romero \& J. Salom, J. (eds.). Cohesión e inteligencia territorial (pp. 105-119). Valencia: PUV / IIDL, Colección Desarrollo Territorial n ${ }^{\circ} 7$.

Eggenberger, M. \& Partidàrio, M. R. (2000). Development of a framework to assist the integration of environmental, social and economic issues in spatial planning. Impact Assessment and Project Appraisal, 18(3), 201-207.

Faludi, A. (2011). Foreword. En J. Farinós (ed. y coord.). De la evaluación ambiental estratégica a la evaluación de impacto territorial. Reflexiones acerca de la tarea de evaluación (pp. 13-16). Valencia: PUV.

Farinós, J. (2005). Nuevas formas de gobernanza para el desarrollo sostenible en el espacio relacional. Ería, (67), 219-235.

Farinós, J. (2007). El reto de una planificación territorial efectiva para poder afrontar los grandes desafíos territoriales en Europa. Documento de discusión presentado a la mesa redonda Los grandes desafíos territoriales en Europa, $V$ Congreso Internacional de Ordenación del Territorio: De los instrumentos programados a la planificación aplicada. Málaga del 22-24 de noviembre, $14 \mathrm{p}$.

Farinós, J. (2008). Gobernanza territorial para el desarrollo sostenible: estado de la cuestión y agenda. Boletín de la Asociación de Geógrafos Españoles, (46), 11-32.

Farinós, J. (ed. y coord.) (2011a). De la evaluación ambiental estratégica a la evaluación de impacto territorial. Reflexiones acerca de la tarea de evaluación. Valencia: PUV.

Farinós, J. (2011b). Inteligencia Territorial para la planificación y la gobernanza democráticas: los observatorios de los territorios. Proyección, V(11), 45-69.

Farinós, J. \& Romero, J. (eds.) (2007). Territorialidad y buen gobierno para el desarrollo sostenible. Nuevos principios y nueva política en el espacio europeo. Valencia: PUV/ IIDL, Colección Desarrollo Territorial $n^{0} 2$.

Farinós, J. \& Romero, J. (2008). La gobernanza como método para encarar los nuevos grandes retos territoriales y urbanos. Boletín de la Asociación de Geógrafos Españoles, (46), 5-9.

Farinós, J., Romero, J. \& Salom, J. (eds.) (2009). Cohesión e inteligencia territorial. Valencia: PUV / IIDL, Colección Desarrollo Territorial n ${ }^{\circ} 7$.

Ferrao, J. (2010). A Geographer at Work Report of a Journey to the World of Political DecisionMaking. IGU Applied Geography Commission. Ericeira, Portugal, 18-22 July, 6 pp.

Geertz, C. (1973). The interpretation of cultures. New York: Basic Books cop.

Gómez, D., Gómez, M. \& Gómez, M. (2011). Los orígenes de la evaluación ambiental estratégica (EAE) y su relación con la evaluación de impacto territorial (EIT). En J. Farinós, (ed. y coord.), De la evaluación ambiental estratégica a la evaluación de impacto territorial. 
Reflexiones acerca de la tarea de evaluación (pp. 167-210). Valencia: PUV.

Habermas, J. (2003). Teoría de la acción comunicativa. Madrid: Taurus, 2 vols.

Healey, P. (2004). The treatment of space and place in the new strategic spatial planning in Europe. International Journal of Urban and Regional Research, (28), 45-67.

Healey, P. (2006). Relational complexity and the imaginative power of strategic spatial planning. European Planning Studies, 14(4), 525-546.

Healey, P. (2008). Knowledge flows, spatial strategy making, and the roles of academics. Environment and Planning C: Government and Policy, (26), 861-881.

Institute on Governance (2003). Principles for good governance in the 21st Century. Policy Brief, (15), 8 pp. Retrieved from http://iog.ca/publications/iog-policy-brief-no-15-principles-forgood-governance-in-the-21st-century/

Jaeck, E. (2009). Les facteurs clés d'un système d'indicateurs quantitatifs, Sixth European Conference on Evaluation of Cohesion Policy - New Methods for Cohesion Policy Evaluation: Promoting Accountability and Learning. Varsovia, 30 de noviembre, 12 p.

Jessop, B. (2004). Multi-level governance and multi-level metagovernance changes in the European Union as integral moments in the transformation and reorientation of contemporary statehood. In I. Bache \& M. Flinders (eds.) Multi-level governance. (pp. 49-74). Oxford: Oxford University Press.

Madoery, O. (2003). El territorio como factor estratégico de desarrollo. Hacia un espacio de gestión metropolitana en el Gran Rosario. [En línea]. Rosario, Argentina: Comunidad Virtual de Gobernabilidad y Liderazgo (CVG). Desarrollo Humano e Institucional. Recuperado de http://cdi.mecon.gov.ar/biblio/docelec/MU1176.pdf

Marques DaCosta, E. (2011). Indicadores de desenvolvimento e ordenamento do território (Opção). Relatório de Programa. Lisboa: CEG-IGOT-UL, 140 p.

Parejo, T. (2007). Nuevas formas de derecho blando para la ordenación del territorio. Enseñanzas de la Estrategia Territorial Europea. En J. Farinós \& J. Romero (eds.). Territorialidad y buen gobierno para el desarrollo sostenible. Nuevos principios y nuevas políticas en el espacio europeo. (pp. 119-145). Valencia: PUV / IIDL, Colección Desarrollo Territorial $\mathrm{n}^{\mathrm{o}} 2$.

Pascual, J.M. (2007). La estrategia territorial como inicio de la gobernanza democrática: los planes estratégicos de segunda generación. Barcelona: Diputació de Barcelona, Colección Documentos de Trabajo, Serie Desarrollo Económico no 3. 160 p. Recuperado de http:// www.diba.cat/pdfs/05562.pdf,

Pascual, J.M. (2011). El papel de la ciudadanía en el auge y decadencia de las ciudades. El fin del gerencialismo o la recuperación de lo público y sus autores. Valencia: Tirant Lo Blanch.

Pereira, D. (2011). El territorio desde una visión integrada de los instrumentos de evaluación ambiental. Posibilidades y dificultades. En J. Farinós (ed. y coord.), De la evaluación ambiental estratégica a la evaluación de impacto territorial. Reflexiones acerca de la tarea de evaluación. (pp. 57-74). Valencia: PUV. 
Prats, J. (2005a). Modos de gobernación de las sociedades globales. En A. Cerrillo (coord.) La gobernanza hoy: 10 textos de referencia. (pp. 145-172). Madrid: INAP.

Prats, J. (2005b). De la burocracia al management, del management a la gobernanza. Las transformaciones de las administraciones públicas de nuestro tiempo. Madrid: MAP, Instituto Nacional de Administración Pública, Colección Estudios Goberna.

Queirós, M. (2009). Teorias da complexidade: princípios para o ordenamento do territorio. Texto de la tercera ponencia 'Las dimensiones sociales y políticas del Medio Ambiente y la Ordenación del Territorio', XI Coloquio Ibérico de Geografía: 'La perspectiva geográfica ante los nuevos retos de la sociedad y el medio ambiente en el contexto ibérico'. Alcalá de Henares, del 1-4 de octubre, $19 \mathrm{p}$.

Romero, J. \& Farinós, J. (2011). Redescubriendo la gobernanza más allá del buen gobierno. Democracia como base, desarrollo territorial como resultado. Boletín de la Asociación de Geógrafos Españoles, (56), 295-319.

Salet, W. \& Faludi, A. (eds.) (2000). The revival of strategic spatial planning. Amsterdam: Royal Netherlands Academy of Arts and Sciences, KNAW.

Seixas, J. (2008). Dinámicas de gobernanza urbana y estructuras del capital sociocultural en Lisboa. Boletín de la Asociación de Geógrafos Españoles, (46), 121-142.

Serrano, A. (2011). La evaluación de las políticas de impacto territorial. Una reflexión desde la toma de decisiones. En J. Farinós (ed. y coord.). De la evaluación ambiental estratégica a la evaluación de impacto territorial. Reflexiones acerca de la tarea de evaluación. (pp. 211-252). Valencia: PUV.

Sorensen, E. \& Torfing, J. (2009). Making governance networks effective and democratic through metagovernance. Public Administration, 87(2), 234-58.

Swyngedouw, E. (2005). Governance innovation and the citizen: the Janus face of governancebeyond-the-state. Urban Studies, 42(11), 1991-2006.

Vallés, J.M. (2004). Ciencia política. Una introducción. Barcelona: Ariel.

Valenzuela, L. M. \& Soria, J. A. (2011). Observatorios territoriales y urbanos en Europa ¿entidades pasivas o instrumentos operativos para la planificación? Ciudad y Territorio Estudios Territoriales (CyTET), XLIII (168), 243-260.

Wassenhoven, L. (2008). Territorial governance, participation, cooperation and partnership: a matter of national culture? Boletín de la Asociación de Geógrafos Españoles, (46), 53-76.

Recepción: 18 de octubre de 2013

Evaluación: 9 de febrero de 2014

Aprobación: 4 de marzo de 2014 